\title{
Depletion of mesospheric sodium during extended period of pulsating aurora
}

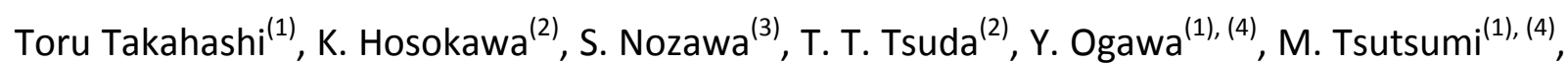
Y. Hiraki ${ }^{(2)}$, H. Fujiwara ${ }^{(5)}$, T. D. Kawahara ${ }^{(6)}$, N. Saito ${ }^{(7)}$, S. Wada ${ }^{(7)}$, T. Kawabata $^{(3)}$, C. Hall $^{(8)}$, and H. Miyaoka ${ }^{(1),(4)}$

(1) National Institute of Polar Research, Tachikawa, Japan

(2) Department of Communication Engineering and Informatics, University of ElectroCommunications, Chofu, Japan

(3) Institute for Space-Earth Environmental Research, Nagoya University, Nagoya, Japan

(4) Graduate University for Advanced Studies (SOKENDAI), Tachikawa, Japan

(5) Faculty of Science and Technology, Seikei University, Musashino, Japan

(6) Faculty of Engineering, Shinshu University, Nagano, Japan

(7) RIKEN Center for Advanced Photonics, Wako, Japan

(8) Troms $\varnothing$ Geophysical Observatory, The Arctic University of Norway, Troms $\varnothing$, Norway

In the last three decades, the impact of auroral particle precipitation on the neutral Na layer has been studied at high latitudes. Recently, we employed simultaneous observations by the sodium lidar and the European Incoherent Scatter (EISCAT) VHF radar at Troms $\varnothing$, Norway $\left(69.6^{\circ} \mathrm{N}, 19.2^{\circ} \mathrm{E}\right)$, to study the effect of auroral particle precipitation on Na density. Our simultaneous observations clearly showed an occurrence of $\mathrm{Na}$ density depletion during auroral ionization. The depletion around $100 \mathrm{~km}$ reached up to $\sim 60 \%$ of the nightly mean $\mathrm{Na}$ density. However, it was still unclear which of the suggested mechanisms dominated the observed Na density depletion.

In this study, we quantitatively evaluated the Na density depletion due to charge transfer reactions between $\mathrm{Na}$ atoms and molecular ions produced by high-energy electron precipitation during a pulsating aurora (PsA). An extended period of PsA was captured by an all-sky camera at the EISCAT radar site during a 2-h interval from 00:00 to 02:00 UT on 25 January 2012. During this period, using the EISCAT VHF radar, we detected three intervals of intense ionization below $100 \mathrm{~km}$ that were probably caused by precipitation of high-energy electrons during the PsA. In these intervals, the sodium lidar at Troms $\varnothing$ observed characteristic depletion of Na density at altitudes between 97 and $100 \mathrm{~km}$. These Na density depletions lasted for $8 \mathrm{~min}$ and represented $5-8 \%$ of the background Na layer. To examine the cause of this depletion, we modeled the depletion rate based on charge transfer reactions with $\mathrm{NO}^{+}$and $\mathrm{O}_{2}{ }^{+}$while changing the $R$ value which is defined as the ratio of $\mathrm{NO}^{+}$to $\mathrm{O}_{2}{ }^{+}$densities, from 1 to 10 . The correlation coefficients between observed and modeled $\mathrm{Na}$ density depletion calculated with typical value $R=3$ for time intervals $T_{1}, T_{2}$, and $T_{3}$ were 0.66 , 0.80 and 0.67 .

The observed $\mathrm{Na}$ density depletion rates fall within the range of modeled depletion rate calculated with $R$ from 1 to 10 . This suggests that the charge transfer reactions triggered by 
the auroral impact ionization at low altitudes is the predominant process responsible for $\mathrm{Na}$ density depletion during PsA intervals.

In this presentation, we will present about these results in detail and future plans for this study by using an upgrade in the data recording and laser control system for the Troms $\varnothing$ sodium lidar. 